\title{
Correction to: Cracking “Open” Technology in Ecohydrology
}

\author{
B. Turner, D. J. Hill, and K. Caton
}

\section{Correction to:}

Chapter 1 in: D. F. Levia et al. (eds.), Forest-Water

Interactions, Ecological Studies 240, https://doi.org/10.1007/978-3-030-26086-6_1

The chapter "Cracking "Open" Technology in Ecohydrology" was previously published as non-open access. It has now been changed to open access under a CC BY-NC-ND 4.0 license and the copyright holder updated to "The Author(s)." The book has also been updated with these changes. 